Gut and Liver, Vol. 13, No. 2, March 2019, pp. 138-139

\title{
Can Statin Prevent the Risk of Colorectal Cancer in Patients with Inflammatory Bowel Disease?
}

\author{
Hyun Joo Jang \\ Department of Gastroenterology, Hallym University School of Medicine, Hwasung, Korea
}

\author{
See, "Statin Exposure Is Not Associated with Reduced Prevalence of Colorectal Neoplasia in Patients with Inflammatory \\ Bowel Disease" by Shailja C. Shah, et al. on page 54, Vol. 13, No. 1, 2019
}

The risk for dysplasia and colorectal cancer (CRC) is increased in patients with long-standing ulcerative colitis (UC) and extensive Crohn's disease (CD). CRC is one of important causes of death in patients with inflammatory bowel disease (IBD). Patients with long-standing UC and colonic CD have about 1- to 6-fold increase in lifetime risk of CRC compared to the general population. ${ }^{1}$ The risk factors for IBD-associated CRC include: prolonged duration of colitis, extent and severity of colitis, coexistence of primary sclerosing cholangitis (PSC), and family history of CRC. ${ }^{2}$ Oncogenic mechanism of IBD-related CRC has been known that chronic or repeated inflammation to the intestinal mucosa leads to the development of CRC via low- and high-grade dysplasia.

There is a controversial issue about whether statins have chemopreventive potentials against CRC, but it still remains a debate. Statins, one of cholesterol-lowering drugs, are used to prevent and treat cardiovascular diseases. Some experimental results recently suggest that statins provide an additional chemopreventive effect by inducing apoptosis, inhibiting angiogenesis, increasing the antitumor effects of several cytokines, and preventing metastasis. ${ }^{3,4}$ Two recent meta-analyses including 40 and 42 individual studies suggested a risk reduction of CRC among statin users. ${ }^{5,6}$

Statins reduce synthesis of cholesterol in liver and reduce the level of serum cholesterol by 3-hydroxy-3-methylglutarylcoenzyme A reductase which is a rate-limiting enzyme in the mevalonate pathway. Its downstream mevalonate products (such as isoprenoid molecules, farnesyl pyrophosphate and geranylgeranyl pyrophosphate) are necessary for post-translational modification and isoprenylation of a variety of proteins such as
Ras and RhoA, which are important for cell growth, cell proliferation, angiogenesis or cell migration. ${ }^{7}$ These proteins are overexpressed in CRC and are associated with tumor invasion. Statin induces apoptosis and suppresses the $\beta$ subunits of insulin-like growth factor 1 receptor (IGF-1R) and IGF-1-induced ERK/Akt activation. ${ }^{3}$ Non HMG-CoA reductase-mediated effects of statins may be through inhibition of inflammation, angiogenesis and cell adhesion, and an antioxidant effect.

There are limited studies investigating the chemopreventive effect of statins against CRC in IBD patients. One study showed that long-term statin use was associated with a reduced risk of both IBD-associated CRC (odds ratio [OR], 0.07; 95\% confidence interval [CI], 0.01 to 0.78) and non-IBD CRC (OR, 0.49; 95\% CI, 0.39 to 0.62$){ }^{8}$ The limitations of this study were recall bias and misclassifications based on self-reporting data. It also did not report disease duration, location of disease, medications, extent of disease and severity. Another recent study of Ananthakrishnan et al. ${ }^{9}$ showed that statin use was associated with about $60 \%$ of risk reduction of CRC in a large cohort including 11,000 IBD patients based on ICD-9 codes.

In previous issue of Gut and Liver, Shah et al. ${ }^{10}$ reported that statin use was not associated with lower rates of dysplasia or CRC in IBD patients undergoing surveillance colonoscopies. There was no significant difference in low-grade dysplasia, high-grade dysplasia or CRC development during follow-up between the statin exposed and non-exposed groups. Propensity score analysis did not change the overall findings. The main strengths of this study are as follows: the results of this study provided detailed information of their IBD history, disease duration, the extent of disease, and histologic activity. This study

Correspondence to: Hyun Joo Jang (https://orcid.org/0000-0003-4424-1968)

Department of Gastroenterology, Hallym University School of Medicine, 7 Keunjaebong-gil, Hwaseong 18450, Korea

Tel: +82-31-8086-2450, Fax: +82-31-8086-2029, E-mail: jhj1229@hallym.or.kr

pISSN 1976-2283 eISSN 2005-1212 https://doi.org/10.5009/gnl19003

@ This is an Open Access article distributed under the terms of the Creative Commons Attribution Non-Commercial License (http://creativecommons.org/licenses/by-nc/4.0) which permits unrestricted non-commercial use, distribution, and reproduction in any medium, provided the original work is properly cited. 
also analyzed statin exposure and occurrence of any dysplasia, and therefore any neoplastic transformation among IBD colitis patients undergoing surveillance colonoscopies for longstanding colitis or PSC-related colitis. However, there are some limitations of this study. This study included insufficient numbers of patients and the low overall rates of dysplasia and CRC to prove differences. The results of this study did not analyze the name, duration and dose of statin. Statins exert different effects depending on cell line, the type of statin being used, concentration of statin, and duration of exposure of cells to statins in experimental environment. The retrospective design of this study also limits to control unmeasured and measured confounders.

In summary, statin use as chemopreventive agents in IBD patients remains controversial. Well-designed and large prospective studies would be needed to prove the chemopreventive role of statin in IBD patients.

\section{CONFLICTS OF INTEREST}

No potential conflict of interest relevant to this article was reported.

\section{REFERENCES}

1. Jess T, Gamborg M, Matzen P, Munkholm P, Sørensen TI. Increased risk of intestinal cancer in Crohn's disease: a metaanalysis of population-based cohort studies. Am J Gastroenterol 2005;100:2724-2729.

2. Stidham RW, Higgins PD. Colorectal cancer in inflammatory bowel disease. Clin Colon Rectal Surg 2018;31:168-178.

3. Jang HJ, Hong EM, Park SW, et al. Statin induces apoptosis of human colon cancer cells and downregulation of insulin-like growth factor 1 receptor via proapoptotic ERK activation. Oncol Lett 2016;12:250-256.

4. Guruswamy S, Rao CV. Multi-target approaches in colon cancer chemoprevention based on systems biology of tumor cell-signaling. Gene Regul Syst Bio 2008;2:163-176.

5. Lytras T, Nikolopoulos G, Bonovas S. Statins and the risk of colorectal cancer: an updated systematic review and meta-analysis of 40 studies. World J Gastroenterol 2014;20:1858-1870.

6. Liu Y, Tang W, Wang J, et al. Association between statin use and colorectal cancer risk: a meta-analysis of 42 studies. Cancer Causes Control 2014;25:237-249.

7. Kobayashi Y, Banno K, Kunitomi H, et al. Is antidyslipidemic statin use for cancer prevention a promising drug repositioning approach? Eur J Cancer Prev. Epub 2018 Nov 8. https://doi. org/10.1097/CEJ.0000000000000497.

8. Samadder NJ, Mukherjee B, Huang SC, et al. Risk of colorectal cancer in self-reported inflammatory bowel disease and modification of risk by statin and NSAID use. Cancer 2011;117:1640-1648.

9. Ananthakrishnan AN, Cagan A, Cai T, et al. Statin use is associated with reduced risk of colorectal cancer in patients with inflammatory bowel diseases. Clin Gastroenterol Hepatol 2016;14:973979.

10. Shah SC, Glass J, Giustino G, et al. Statin exposure is not associated with reduced prevalence of colorectal neoplasia in patients with inflammatory bowel disease. Gut Liver 2019;13:54-61. 